\title{
Is it worth including subtalar joint in ultrasound ankle assessment of patients with juvenile idiopathic arthritis?
}

\author{
Stefano Lanni ${ }^{1 *}$, Francesca Bovis ${ }^{1}$, Francesca Magnaguagno ${ }^{1}$, Angelo Ravelli ${ }^{1,2}$, Stefania Viola ${ }^{1}$, \\ Annette Von Scheven-G区te ${ }^{1}$, Nicola Ruperto ${ }^{1}$, Alberto Martini ${ }^{1,2}$, Clara Malattia 1,2 \\ From 21st European Pediatric Rheumatology (PReS) Congress \\ Belgrade, Serbia. 17-21 September 2014
}

\section{Introduction}

The ankle is a complex anatomical structure owing to the multiple joint recesses and surrounding tendons. The involvement of subtalar joint (STJ) can be difficult to discern clinically from tibiotalar, tarsal or adjacent tendon disease. To date, only a few efforts have been centred around ultrasound (US)-detectable assessment of STJ in juvenile idiopathic arthritis (JIA).

\section{Objectives}

1) To assess the frequency of US-detectable involvement of STJ, 2) To compare clinical versus US assessment in STJ detection, 3) To determine the most informative scanning approach to STJ.

\section{Methods}

Fifty consecutive JIA patients, followed at the study center, with clinically-detected ankle involvement were enrolled. If both the ankles were involved, only the most affected side was selected for US. All clinical and US examinations were performed at the same day by experienced physicians and ultrasonographers, respectively, blinded to each other evaluations. US findings were collected using a lateral, medial and posterior STJ scanning approach. US synovitis was considered when both or either of joint effusion and synovial hypertrophy, with or without power Doppler signal, were visualized. Inter-observer reliability of US STJ involvement was tested using Cohen's kappa coefficient in a subgroup of 24 patients. A control group of 10 healthy subjects was recruited.

\section{Results}

None of the controls showed US STJ synovitis. US detected synovitis in 27 (54\%) STJs of patients. Agreement between clinical and US assessment for presence and absence of STJ involvement was found in 17 (34\%) and $16(32 \%)$ ankles, respectively. In 10 (20\%) STJs not considered to be clinically affected, synovitis was found on US. In 7 (14\%) ankles labelled as having STJ involvement on clinical examination US was negative for STJ, but showed the involvement of different anatomical sites (midfoot, tibiotalar joint, tendons). Overall, the concordance between clinical and US evaluation was poor $(\mathrm{k}=0.32)$. The Cohen-kappa value for inter-observer reliability of STJ involvement on US was high $(\mathrm{k}=0.92)$. All patients having US findings in the medial and/or posterior side of STJ presented also with US findings using the lateral scanning approach, but the reverse was not true.

\section{Conclusion}

US is more sensitive than clinical evaluation in the assessment of STJ in ankles with active disease. The high frequency of its involvement may suggest to include the assessment of STJ in US scanning protocols. In this perspective, the lateral approach to the joint seems to be more appropriate for US evaluation of STJ involvement.

\section{Disclosure of interest}

None declared.

${ }^{1}$ Irccs Istituto Giannina Gaslini, Genova, Italy

Full list of author information is available at the end of the article 


\title{
Authors' details
}

'Irccs Istituto Giannina Gaslini, Genova, Italy. Universita' di Genova, Genova,

Italy.

Published: 17 September 2014

\author{
doi:10.1186/1546-0096-12-S1-P36 \\ Cite this article as: Lanni et al:: Is it worth including subtalar joint in \\ ultrasound ankle assessment of patients with juvenile idiopathic \\ arthritis? Pediatric Rheumatology 2014 12(Suppl 1):P36.
}

Submit your next manuscript to BioMed Central and take full advantage of:

- Convenient online submission

- Thorough peer review

- No space constraints or color figure charges

- Immediate publication on acceptance

- Inclusion in PubMed, CAS, Scopus and Google Scholar

- Research which is freely available for redistribution

Submit your manuscript at www.biomedcentral.com/submit

() BioMed Central 